\title{
AS TEORIAS DE CARTEIRA DE MARKOWITZ E DE SHARPE: \\ UMA APLICAÇÃO NO MERCADO \\ BRASILEIRO DE AÇÕES ENTRE \\ JULHO/95 E JUNHO/200O
}

FRANCISCO ANTÔNIO MESQUITA ZANINI

Professor da Universidade do Vale do Rio dos Sinos - UNISINOS Av. Unisinos, 950 - São Leopoldo/RS - CEP 93022-000

E-mail: fzanini@unisinos.br

ANTONIO CARLOS FIGUEIREDO

Professor da Pontifícia Universidade Católica do Rio de Janeiro IAG - Instituto de Administração e Gerência Rua Marquês de São Vicente, 225 - Gávea - Rio de Janeiro/RJ - CEP 22453-900 E-mail: figueiredo@iag.puc-rio.br 


\section{RESUMO}

Questão central para analistas e administradores de carteiras de investimento, o gerenciamento do risco é um desafio permanente também para o meio acadêmico. Markowitz apresentou, em I952, as bases da Moderna Teoria de Carteiras. Dadas as dificuldades técnicas para aplicação da Teoria de Markowitz àquela época, Sharpe desenvolveu, já nos anos 60, uma Teoria baseada no modelo original, mas adotando expressivas simplificações, que facilitavam em muito os cálculos necessários à sua implementação. O cerne do presente trabalho é a comparação entre o desempenho de uma e de outra Teoria. Procura-se identificar se as simplificações adotadas por Sharpe prejudicam ou não o desempenho de seu modelo. Adicionalmente discutem-se eventuais benefícios para o investidor, decorrentes da aplicação das duas Teorias.

\section{PALAVRAS-CHAVE}

Teorias de carteira; Risco; Administração do risco.

\section{ABSTRACT}

Risk management is a crucial question and a challenge both to portfolio analysts and managers and to the academy. In I952, Markowitz has presented the basis a what would be known as the Modern Portfolio Theory. In the next decade, based on Markowitz's studies, Sharpe has developed a simplified model, called Single Index Model, whose application, because of the adoption of some simplification premises, became easier through a considerable reduction of the volume of required calculations. This work seeks to determine if the simplifications proposed by Sharpe's model do significantly affect its results or, more specifically, if the portfolio optimization using both models - Markowitz's original model and Sharpe's Single Index Model - gives significantly different results.

\section{KEYWORDS}

Portfolio theory; Risk; Risk management. 


\section{INTRODUÇÃO}

Vaughan (I997) observa que, nos dias atuais, a questão da administração do risco está presente na vida de todos. Para o autor a história da espécie humana é uma cronologia de exposições ao infortúnio e às adversidades, e de esforços para conviver com eles. A continuidade da história humana, então, é uma testemunha do sucesso de nossos ancestrais em administrar o risco.

No que tange ao processo de alocação da riqueza, a análise e o domínio do risco na gestão de investimentos, são desafios permanentes para o meio acadêmico e para os administradores de ativos que atuam nos mercados financeiro e de capitais. Bruni e Famá (I998) afirmam que um dos primeiros acadêmicos a considerar a importância do risco na gestão de ativos foi Harry Markowitz, em artigo clássico - Portfolio Selection.

De uma forma simplificada, pode-se dizer que o modelo proposto por Markowitz (I952), preconiza que o retorno esperado para um conjunto de ativos é a média ponderada dos retornos esperados para cada ativo individual. Já o risco deste conjunto de ativos não é a média dos riscos dos ativos individuais, mas uma função das variâncias individuais de cada ativo e de uma parcela das covariâncias entre os ativos, calculadas dois a dois. A dificuldade existente para a aplicação da teoria proposta por Markowitz naquela época, dado o elevado número e certa complexidade dos cálculos necessários ao seu desenvolvimento, levou Sharpe a desenvolver um modelo simplificado, chamado Modelo de Índice Único - MIU.

Em seu modelo, Sharpe (ig63), diferentemente da proposição de Markowitz, não parte do princípio que os retornos entre os ativos estão correlacionados entre si, mas sim com um índice único, este representativo do retorno de todo o mercado onde são transacionados aqueles ativos. Com este pressuposto, o número de cálculos a serem efetuados cai sensivelmente, mesmo que a custo de eventual perda de precisão, dadas as simplificações introduzidas.

O objetivo central deste trabalho é determinar se existe diferença no desempenho de carteiras de ativos quando aplicados o modelo original de Markowitz e o Modelo de Índice Único de Sharpe no mercado de ações brasileiro. Adicionalmente são propostas também duas outras questões:

40 - É válida a aplicação das Teorias de Carteira de Markowitz - MM, e de Sharpe, o Modelo de Índice Único - MIU, no mercado brasileiro de ações, ou seja, sua aplicação traria benefícios ao investidor?

- Quais os períodos de tempo (janelas móveis) são os mais adequados para utilizar como base de dados para a otimização das carteiras?

Para cumprir com os objetivos propostos, inicia-se realizando uma revisão sobre a fundamentação teórica dos dois modelos. Discute-se, em seguida, uma 
maneira de medir de forma conjunta o risco e o retorno de carteiras de ativos, assim como a adoção de parâmetros adicionais para avaliação de carteiras. A seguir são apresentados os procedimentos metodológicos da presente pesquisa, bem como os resultados alcançados. Finalmente apresentam-se as conclusões do trabalho.

\section{OS DOIS MÉTOdOS REVOLUCIONÁRIOS NA GESTÃO dE CARTEIRAS}

\subsection{MARKOWITZ: O INÍCIO DA MODERNA TEORIA DE PORTFÓLIOS - MTP}

Markowitz (I952) revolucionou os meios acadêmicos de sua época no que diz respeito à análise de carteiras de investimento. Em contraposição ao pensamento dominante à época, de que a melhor situação para a alocação de recursos era a concentração dos investimentos nos ativos que ofereciam os maiores retornos esperados, Markowitz demonstra que seria possível obter combinações mais eficientes de alocação de recursos, com melhor relação retorno esperado versus risco incorrido.

As premissas fundamentais assumidas pelo autor para a constituição da base do que passou a se chamar de A Moderna Teoria de Portfólios - MTP - foram as seguintes:

I) os investidores avaliariam as carteiras apenas com base no retorno esperado e no desvio padrão dos retornos sobre o horizonte de tempo de um período;

2) os investidores seriam avessos ao risco. Se instados a escolher entre duas carteiras de mesmo retorno, sempre escolheriam o de menor risco;

3) os investidores estariam sempre insatisfeitos em termos de retorno. Instados a escolher entre duas carteiras de mesmo risco, sempre escolheriam a de maior retorno;

4) seria possível dividir continuamente os ativos, ou seja, ao investidor seria permitido comprar mesmo frações de ações;

5) existiria uma taxa livre de risco, à qual o investidor tanto poderia emprestar quanto tomar emprestado (premissa não adotada no presente estudo, que trabalhou apenas com ações, além de não permitir vendas a descoberto);

6) todos os impostos e custos de transação seriam considerados irrelevantes;

7) todos os investidores estariam de acordo em relação à distribuição de probabilidades das taxas de retorno dos ativos. Isto significa que somente existiria um único conjunto de carteiras eficientes.

Partindo destas premissas, Markowitz (I952) determina as duas características fundamentais de uma carteira: o seu retorno esperado e a sua variância, esta 
última representando o risco da carteira. A primeira característica da carteira, seu retorno esperado, é simplesmente a média ponderada dos retornos dos ativos individuais que o compõe, conforme a seguir:

$$
\bar{R}_{p}=\sum_{i=1}^{N} \mathrm{X}_{i} \mathrm{E}\left(R_{i}\right) \quad(\mathrm{I}), \text { onde: } \quad \begin{aligned}
& \text { i é o percentual investido no ativo i; } \\
& \left(R_{\mathrm{i}}\right) \text { é o retorno esperado do ativo } i .
\end{aligned}
$$

A segunda característica fundamental de uma carteira é o seu risco, medido pela sua variância:

$$
\sigma_{p}^{2}=\mathrm{E}\left(R_{p}-\bar{R}_{p}\right)^{2}
$$

Esta fórmula pode ser também representada de forma mais interessante em termos práticos, em especial quando o propósito do estudo é a construção de carteiras. Esta forma é a partir das variâncias dos ativos individuais e das covariâncias entre todos os ativos, calculadas dois a dois. Sua conhecida derivaçao pode ser encontrada em Elton e Gruber (I995), e seu resultado final é:

$$
\sigma_{p}^{2}=\sum_{j=1}^{N} \mathrm{X}_{j}^{2} \sigma_{j}^{2}+\sum_{j=1}^{N} \sum_{\substack{k=1 \\ k \neq j}}^{N} \mathrm{X}_{j} \mathrm{X}_{k} \sigma_{j k}
$$

\subsection{O MODELO DE MARKOWITZ E OS EFEITOS DA DIVERSIFICAÇÃO}

Uma das maiores contribuições dos estudos de Markowitz foi ressaltar a importância da diversificação, conceito contestado por importantes acadêmicos de então, como Keynes. O conceito da diversificação decorre da constatação de que os preços dos ativos financeiros não se movem de modo exatamente conjunto. Ou, dizendo de outra forma, eles têm uma correlação imperfeita. Nesta condição, a variância total de uma carteira é reduzida pelo fato de que a variação no preço individual de um ativo ser compensada por variações complementares nos demais.

A Gráfico I, adiante, mostra que: quando a correlação é perfeitamente positiva, não há benefício na diversificação; quando a correlação é perfeitamente negativa, obtém-se o melhor benefício possível na diversificação; quando a correlação fica no intervalo entre -I e +I, obtém-se também algum benefício. Isto pode ser também visualizado quando se substitui a covariância entre os ativos $j$ e $k$ na equação correspondente pelo produto dos desvios padrão dos mesmos ativos e sua correlação, conforme a seguir:

$$
\sigma_{p}^{2}=\sum_{j=1}^{N} \mathrm{X}_{j}^{2} \sigma_{j}^{2}+\sum_{j=1}^{N} \sum_{\substack{k=1 \\ k \neq j}}^{N} \mathrm{X}_{j} \mathrm{X}_{k} \rho_{j k} \sigma_{j} \sigma_{k}
$$


Considerando que não haverá desvio padrão negativo, nem $\mathrm{X}_{\mathrm{i}}$ negativo, pois não se está considerando a possibilidade de vendas a descoberto, é fácil observar que o segundo termo da equação fica negativo quando a correlação é negativa, diminuindo o valor total do risco da carteira.

Elton e Gruber (I995) demonstram o formato que toma a fronteira eficiente, quando combinam-se dois ativos com correlação perfeitamente positiva $\left(\rho_{\mathrm{I} 2}=\mathrm{I}\right)$, perfeitamente negativa $\left(\rho_{\mathrm{I} 2}=-\mathrm{I}\right)$, e correlação dentro deste intervalo $\left(-\mathrm{I}<\rho_{\mathrm{I} 2}<\mathrm{I}\right)$. Observe-se a Gráfico I a seguir:

\section{GRÁFICO I}

\section{RISCO DA CARTEIRA PARA DIFERENTES VALORES DE CORRELAÇÃO ENTRE DOIS ATIVOS}

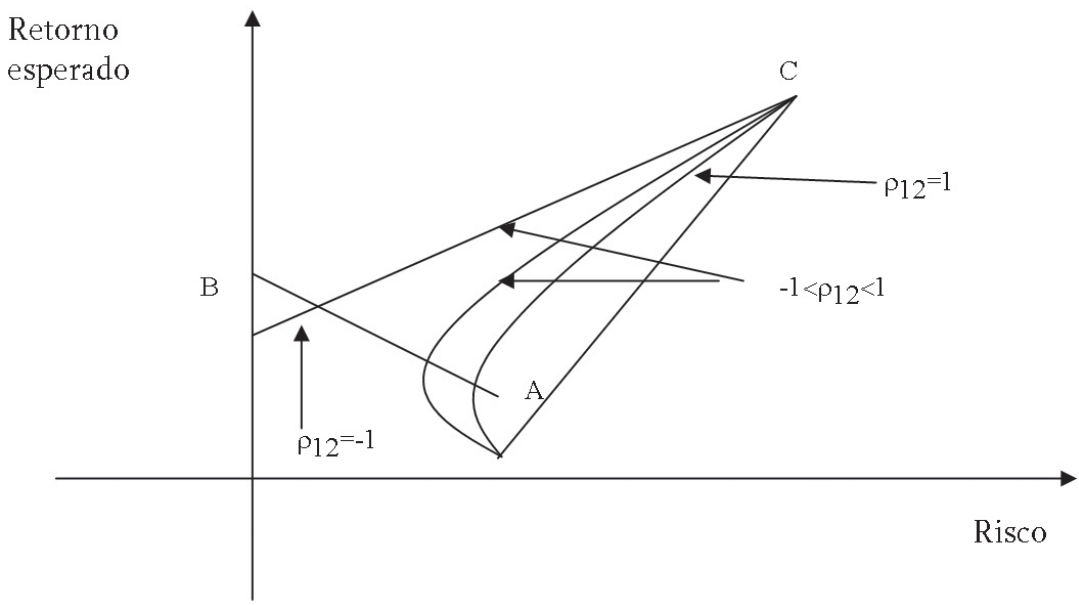

\subsection{O MODELO DE ÍNDICE ÚNICO DE SHARPE - MIU}

O modelo precedente, de Markowitz, necessita elevadíssimo número de informações para ser aplicado. Bruni e Famá (I998) citam um trabalho de Sharpe, datado de I96I, que determinou que o melhor computador IBM existente ao início daquela década necessitaria de cerca de 33 minutos para realizar uma otimização de uma carteira composta por cem ativos, e isto a um elevado custo de US\$300, o que inviabilizava testes e simulações.

Esta dificuldade de operacionalização do modelo original de Markowitz levou o mesmo Sharpe (1963) a desenvolver um modelo alternativo, bastante mais simples, em especial no que concerne ao número de inputs necessários, o chamado Modelo de Índice Único - MIU. A suposição básica de Sharpe ao criar o modelo, não era a de que os retornos entre os ativos estariam correlacionados entre si, mas sim com um índice único, este representativo dos retornos de todo 
o mercado onde transacionados. Para este modelo, ao se relacionar os retornos de cada ativo com o retorno do mercado, estar-se-á, indiretamente, relacionando os retornos dos ativos entre si.

Elton e Gruber (I995) derivam o modelo de Sharpe a partir da equação básica para o retorno do ativo $i$ e demais suposições:

$$
R_{i}=a_{i}+\beta_{i} R_{m}
$$

onde: $a_{i}$ é o componente do retorno do ativo $i$ que é independente da performance do mercado, variável aleatória;

$R_{m}$ é a taxa de retorno do mercado, variável aleatória;

$\beta_{i}$ é constante, que mede a expectativa de mudança no retorno do ativo $i$ dada uma mudança no retorno de mercado $\left(R_{m}\right)$.

Esta equação básica divide o retorno de um ativo em duas partes. Uma parte indexada ao mercado, e outra parte independente do mercado. O termo $a_{i}$ representa a parcela do retorno do ativo que é independente do mercado. Sharpe (1963), para simplificar seu modelo, supoe:

I) que $e_{i}$, o erro padrão da estimativa da reta de regressão, tem valor esperado igual a zero;

2) que $e_{i}$ e $R_{m}$ não sao correlacionados;

3) e que $e_{i}$ é independente de $e_{j}$ para quaisquer ativos $i$ e $j$.

A partir destas suposições, Sharpe (1963) deriva então as três equações representativas do retorno esperado, variância do retorno e covariância entre os retornos entre os ativos $i$ e $j$, cujos resultados finais são, respectivamente:

$$
\bar{R}_{i}=\alpha_{i}+\beta_{i} \bar{R}_{m} \text { (5) } \quad \sigma_{i}^{2}=\beta_{i}^{2} \sigma_{m}^{2}+\sigma_{e i}^{2}(6) \quad \sigma_{i j}=\beta_{i} \beta_{j} \sigma_{m}^{2} \quad \text { (7) }
$$

A partir destes resultados, voltando-se à equação fundamental para retorno esperado de uma carteira de ativos e substituindo-se pelos termos derivados pelo modelo de Sharpe, tem-se:

$$
\bar{R}_{p}=\sum_{i=1}^{N} \mathrm{X}_{i} \alpha_{i}+\sum_{i=1}^{N} \mathrm{X}_{i} \beta_{i} \bar{R}_{m}
$$

Da mesma forma, voltando-se à equação fundamental para variância de um portfólio de ativos, substituindo-se pelas equações derivadas pelo modelo de Sharpe, e fazendo-se as devidas simplificações:

$$
\sigma_{p}^{2}=\beta_{p}^{2} \sigma_{m}^{2}+\sum_{i=1}^{N} \mathrm{X}_{i}^{2} \sigma_{e i}^{2}
$$




\section{A questão da RELAÇÃo RetORNO OBTIDO VERSUS RISCO INCORRIDO}

A simples obtenção de retorno mais elevado num investimento não é prova cabal e verdadeira de sua superioridade em relação a outro. Em verdade o que se quer determinar é se o investimento proporcionou um retorno adequado ao seu nível de risco. Como afirma Ellis (2000:I7):

A questão primordial para um administrador de investimentos é: Como você usa produtivamente o risco e a ausência dele? Como você os faz trabalharem para você e para seus clientes?

Passa-se a discutir um bom parâmetro para esta avaliação.

Elton e Gruber (I995), Jacob (2000) e Sá (I999) apresentam uma medida de desempenho ajustada pelo risco, bastante interessante e que é praticamente padrão em análises da relação risco/retorno de carteiras de investimento. É o índice de avaliação proposto por Sharpe e que leva seu nome. O índice de Sharpe $\left(\mathrm{I}_{\text {sharpe }}\right)$ é fundamentado na teoria do CAPM, a partir do estudo da chamada Capital Market Line - CML, e é determinado pela expressão:

$$
I_{\text {Sharpe }}=\frac{\bar{R}_{p}-R_{F}}{\sigma_{p}}
$$

$\mathrm{O} \mathrm{I}_{\text {sharpe }}$ expressa a rentabilidade média excedente por unidade de risco total da carteira. A rentabilidade média excedente é a diferença entre a rentabilidade média da carteira sob análise e a rentabilidade proporcionada pelo ativo sem risco. Em nosso mercado existe variação acentuada na rentabilidade proporcionada pelo ativo livre de risco, o que determina trabalhar-se aqui com o retorno esperado $-\bar{R}_{F}$, média dos retornos do período, critério utilizado, dentre outros, por Bruni e Famá (I998) e Brochmann e outros (2000). Adota-se como $\bar{R}_{F}$ a Taxa Média Selic - TMS.

Importa destacar o fato de que o $\mathrm{I}_{\text {sharpe }}$ é uma média e como tal deve ser tratado ao se realizar comparações de desempenho entre diversas carteiras. Ferraz e Taciro Júnior (2000) chamam a atenção para o fato de que muitas vezes as comparações de $\mathrm{I}_{\text {sharpe }}$ são feitas de forma inadequada, sem a necessária consideração de que se trata de um índice médio.

\section{UM OUTRO PARÂMETRO PARA AVALIAÇÃO}

Uma questão que se coloca também como objetivo deste trabalho refere-se à validade ou não de utilização destes modelos no mercado brasileiro. Muitos estu- 
dos têm demonstrado que mesmo diversificações ingênuas podem ser bastante eficientes. Evans e Archer (I968) chegaram à conclusão que uma carteira não deve conter mais do que io ativos, uma vez que o impacto marginal na variância da mesma, em decorrência do acréscimo de um iıffl ativo é desprezível.

Fischer e Lorie (I970), estudando os efeitos da diversificação no mercado norte americano no período compreendido entre 1926 e i965, determinaram que estratégias de investimento completamente aleatórias e com número de oito ativos não diferiram significativamente em desempenho de carteiras contendo número maior de ações. Há que se considerar, contudo, as possíveis limitaçoes do citado trabalho. En Chen e Lee (I98I) demonstra-se que prazos de tempo muito dilatados e um número de ativos não muito grandes podem tornar os resultados viesados. De forma geral, eles recomendam trabalhar com um grande número de ativos e prazos não muito dilatados, ainda que não especifiquem que prazos seriam estes.

Para o mercado brasileiro, Chára et al. (I998) demonstraram, em um estudo que apanhou um período de dois anos - abril de 1996 a abril de I998, que uma carteira aleatória qualquer, formado por I4 dentre 187 ações negociadas na Bolsa de Valores de São Paulo, teria apresentado retorno superior à média de todos os fundos de investimento em ações que permaneceram ativos durante todo o período, sem, no entanto, incorrer em maior risco.

Este trabalho adotará também uma carteira com diversificação ingênua para verificar seu desempenho em termos de risco e retorno, comparando-o com os mesmos parâmetros obtidos pelas carteiras otimizadas com a aplicação do MM e do MIU. Isto permitirá testar a validade ou não da aplicação de tais teorias no mercado brasileiro. Adotar-se-á uma carteira com I4 ativos, mesmo número com que Chára et al. (1998) trabalharam.

\section{ESTUDOS ANTERIORES}

O próprio Sharpe (i963), no trabalho em que lança a proposta do MIU, diz que a alocação dos ativos conforme o seu modelo é praticamente idêntica àquela obtida com o modelo original de Markowitz, mas o autor só mostra o resultado da alocação dos ativos, não o resultado do desempenho das carteiras. Ali Sharpe trabalhou com dois grupos de vinte ativos no mercado norte-americano, por um período de doze anos, de I940 até I95I, mas não explicita exatamente quais premissas adotou.

Elton, Gruber e Urich (I978) realizaram um estudo com certa semelhança com o atual, para o mercado norte americano. Naquele estudo, surpreendentemente segundo os autores, o modelo de Markowitz teve desempenho inferior ao Modelo de Índice Único, apesar deste último ter sido criado exatamente com o intuito de simplificar o primeiro, e, com isto, em princípio, perdendo informações. Em seu 
trabalho, realizado para um período de cinco anos e com roo ativos, Elton, Gruber e Urich estimaram o beta dos ativos de quatro formas diferentes, e o seu desempenho para a predição das matrizes de correlação entre os ativos em todas elas foi significativamente superior à realizada com o uso do Modelo de Markowitz.

Brochmann e outros (2000) realizaram um estudo para o mercado brasileiro, limitado ao ano de I999 e com apenas io ativos. Naquele trabalho, foi utilizada uma única janela móvel (de I2 meses) para a otimização das carteiras, com os dados históricos de retornos dos ativos sob otimização. Os resultados indicaram que o Modelo de Markowitz mostrou-se mais eficiente do que o modelo proposto por Sharpe.

Uma vez que não há estudo comparativo dos dois modelos com uma série mais longa de dados e com um maior número de ativos para o mercado brasileiro de ações, entende-se relevante uma nova comparação do desempenho em nosso mercado acionário.

Há que se considerar, ainda, que no presente estudo foram formadas carteiras a partir de vários prazos - janelas móveis - para a obtenção dos retornos históricos dos ativos, e, a partir daí determinar as fronteiras eficientes destas carteiras. Bruni e Famá (I998) em um estudo que avaliou o desempenho no mercado brasileiro de uma carteira com diversificação ingênua e outra otimizada de acordo com a teoria proposta por Markowitz, trabalharam com três prazos para esta janela móvel - I2, 24 e 36 meses, concluindo pela superioridade da janela de I2 meses. No presente trabalho pretendeu-se adicionar novos pontos a esta curva, estabelecendo-se janelas móveis também para outros prazos, diferentes daqueles.

Hieda e Oda (1998) também realizaram trabalho semelhante ao de Bruni e Fama, com o uso apenas do modelo de Markowitz. Mas as otimizações foram realizadas tendo como parâmetros as valorizações diárias dos vinte principais ativos do Ibovespa, e janelas móveis de um quadrimestre, prazo de vigência daquele índice. Além disto, a otimização das carteiras foi realizada tendo como parâmetro a maximização do Índice de Sharpe, um critério diferente do estudo de Bruni e Fama (I998) e de Brochmann e outros (2000).

\section{METODOLOGIA}

Otimização das carteiras: Os trabalhos empíricos nesta área utilizam dados históricos para estimar os retornos e riscos esperados, como forma de evitar a subjetividade e dificuldade de se fazer previsões. Este é também o critério aqui utilizado, o que significa dizer que se adota a premissa de que o passado é relevante na determinação do futuro. Foram formadas séries de dados com retornos históricos, com horizontes de tempo de 6, 9, I2, I5 e I8 meses anteriores ao mês sob otimização. Os dados dos retornos dos ativos componentes da amostra e 
do Ibovespa, no período de janeiro de 1994 até junho de 2000 foram obtidos da base dados da Economática. Trabalhou-se com dados já deflacionados pelo IGPDI. Os dados da Taxa Média Selic e do IGP-DI foram obtidos de banco de dados do Banco do Brasil S.A. A partir dos 20 principais ativos componentes do Ibovespa (em média $89,80 \%$ do total do índice no período), realizou-se a otimização das carteiras conforme o MM ou MIU, com o auxílio do recurso Solver, do software MS Excel. O procedimento realizado para a otimização das carteiras foi o seguinte:

I) no mês t (junho de I995) calculou-se todos os dados necessários à entrada nas equações para risco e retorno de cada um dos modelos;

2) com o auxílio do Solver encontrou-se a composição da carteira de mínima variância dentre todas as combinações possíveis, incluídas as restrições e premissas utilizadas no presente trabalho: $X_{i} \geq 0 \forall X_{i}$, que significa a inexistência de alavancagem, e $\sum \mathrm{X}_{i}=1$, significando que todos os recursos serão investidos;

3) calculados os percentuais de participação de cada ativo calculou-se o retorno da carteira efetivamente obtido no mês subseqüente, $t+1$, jul/I995. A seguir os procedimentos são repetidos, com a inclusão dos retornos dos ativos no próprio mês t+I, ao mesmo tempo em que são eliminados os dados referentes aos retornos do primeiro mês de cada série. Roda-se novamente o recurso Solver para nova otimização, formando a nova carteira de mínima variância, a vigorar no mês $t+2$, e assim sucessivamente até $o$ mês $t+60$, junho de 2000.

O critério de obtenção da carteira de variância mínima foi adotado uma vez que não é possível determinar a priori qual o retorno que se quer obter ou qual o risco que se quer correr, dado que se trabalha com dados históricos. Pode-se, por exemplo, desejar um nível de retorno ou de risco que os dados históricos mostram inexeqüível.

Montou-se a carteira com diversificação ingênua com I4 ativos obtidos aleatoriamente dentre os mesmos 20 principais integrantes do Ibovespa. Utilizou-se o recurso "Aleatório entre ( )" do software MS Excel. Alterou-se a carteira somente quando algum de seus I4 ativos deixou de figurar dentre os 20 principais do Ibovespa.

Testes estatísticos: A partir dos retornos médios e desvios padrão dos retornos das carteiras, e dos retornos do ativo livre de risco, calculou-se os Índices de Sharpe e os desvios padrão deste Índice de todas as carteiras. Com estas estatísticas básicas, procederam-se diversos testes estatísticos com o fito de elucidar a questão central da presente pesquisa, verificar se as diferenças de desempenhos entre as carteiras são significantes, ou seja, se não podem ser atribuídas unica- 
mente ao acaso. Todos os testes foram estabelecidos com um intervalo de confiança de $95 \%$ e foram realizados com o software SPSS, versão 9.0 profissional, ou com o auxílio de planilhas Excel.

Teste preliminar: Foi realizado um teste preliminar, o teste de normalidade de Kolmogorov-Smirnov. Este é um teste não paramétrico, realizado com o intuito de verificar se a distribuição dos resultados das amostras é aproximadamente Normal, premissa básica adotada pelas teorias de carteira de Markowitz e de Sharpe, além de ser hipótese exigida para a realização de alguns testes paramétricos. O teste consiste na transformação da distribuição de retornos encontrada, em uma distribuição assintótica, que é então comparada com uma distribuição normal padronizada. A comparação é realizada com o uso da estatística $z$ crítica para o intervalo de confiança considerado.

Testes para comparação de desempenho: os testes para comparação de desempenho das carteiras foram divididos em dois conjuntos de procedimentos. O primeiro conjunto de testes foi realizado com o intuito de determinar eventual diferença de desempenho entre as carteiras otimizadas de acordo com as Teorias de Markowitz e de Sharpe, uma versus a outra, ou seja, são testes entre dois conjuntos de observações.

O segundo conjunto de testes foi realizado com o intuito de comparar o desempenho das carteiras otimizadas de acordo com uma e com outra Teoria separadamente, procurando identificar se determinado prazo de dados históricos utilizados na otimização (I8, I5, I2, 9 e 6 meses) conduz a resultados significativamente superiores ao outro. Estes grupos de carteiras também foram ampliados, com a introdução na comparação múltipla, de uma carteira com diversificação ingênua e da carteira de mercado (Ibovespa).

a) Comparação Markowitz versus Sharpe: este primeiro conjunto de testes foi dividido em dois procedimentos distintos: I) os testes realizados para comparar o desempenho das médias de retornos de carteiras, e, 2) os testes realizados para comparar o desempenho dos Índices de Sharpe das carteiras.

aI) Comparação dos desempenhos das carteiras pelas suas médias de retornos: o primeiro procedimento realizado foi o teste de duas amostras para média, teste $t$. Conforme Stevenson (I98I) e Meyer (I972), os testes de duas amostras são utilizados para decidir se as médias de duas populações são iguais, como por exemplo, para comparar dois métodos de ensino ou duas marcas, adequando-se ao presente estudo, para a análise da existência de eventual diferença de médias de desempenhos dos retornos. A estatística $t$ teste é comparada com a estatística $t$ crítica para determinação de eventual diferença significativa entre as médias.

Os testes foram realizados da seguinte forma: i) Markowitz versus Sharpe, dois a dois, ou seja, os resultados dos retornos da carteira otimizada com dados históricos de I8 meses utilizando o Modelo de Markowitz, contra os mesmos 
resultados utilizando o Modelo de Sharpe, com os mesmos dados históricos de I8 meses, e assim sucessivamente para as carteiras otimizadas com dados históricos de I5, I2, 9 e 6 meses, dois a dois; ii) Markowitz versus Sharpe global, ou seja, as cinco médias dos resultados de um modelo versus as cinco médias dos resultados obtidos com o outro.

a2) Comparação de desempenho das carteiras pelos seus Índices de Sharpe: o segundo conjunto de procedimentos para a comparação de desempenho das carteiras, agora tendo como parâmetro os seus Índices de Sharpe, foi idêntico ao anterior com relação ao objetivo, testar Markowitz versus Sharpe, porém, diferente no que se refere ao teste estatístico utilizado, este um teste específico para tal comparação. As propriedades do Índice de Sharpe amostral foram estudadas, dentre outros, por Chen e Lee (I98I). Os testes estatísticos utilizados para a comparação das carteiras são aqueles propostos por Jobson e Korkie (I98I) e utilizam basicamente teoria assintótica, que busca reproduzir como as estatísticas se comportam quando o tamanho da amostra tende ao infinito. Para a comparação de dois fundos ou carteiras, Jobson e Korkie (I98I) demonstram que os estimadores da distribuição assintótica do índice de Sharpe são Normais, com média e variância dadas, aproximadamente, por:

$$
\begin{gathered}
E\left(S \hat{h}_{i n}\right) \approx\left(\sigma_{n} \mu_{i}-\sigma_{i} \mu_{n}\right)\left(1 \quad \frac{1}{4 T}+\frac{1}{32 T^{2}}\right) \\
\hat{\Theta}=\frac{1}{T}\left[2 \sigma_{i}^{2} \sigma_{n}^{2}-2 \sigma_{i} \sigma_{n} \sigma_{i n}+\frac{1}{2} \mu_{i}^{2} \sigma_{n}^{2}+\frac{1}{2} \mu_{n}^{2} \sigma_{i}^{2}-\frac{\mu_{i} \mu_{n}}{2 \sigma_{i} \sigma_{n}}\left(\sigma_{i n}^{2}+\sigma_{i}^{2} \sigma_{n}^{2}\right)\right] \\
\text { A estatística z teste é dada então por } z_{\text {teste }}=\frac{S \hat{h}_{i n}}{\sqrt{\hat{\Theta}}}
\end{gathered}
$$

A estatística teste é então comparada com uma estatística $z$ padronizada. Se a estatística teste é maior do que a estatística crítica, temos que a diferença entre as médias é significativa, ou seja, não pode ser considerada como decorrente de desvios aleatórios da amostra.

b) Comparação entre os prazos utilizados para otimização: observe-se que além de testar o desempenho das carteiras otimizadas com um e outro modelo, também se quer testar se algum dos prazos utilizados para a otimização é consistentemente superior ao outro. Quase instintivamente pode-se pensar em expandir os testes t ou $z$ para as carteiras otimizadas de acordo com um, e, depois, com o outro modelo, duas a duas, obtendo-se $n-1$ testes independentes. No entanto, como chamam a atenção Ferraz e Taciro Júnior (2000), este procedimento infla 
de forma significativa a probabilidade de erro tipo I, ou seja, a probabilidade de se rejeitar a hipótese de igualdade de médias, quando ela é verdadeira.

Nesta condição de teste, considerando-se que a probabilidade de erro tipo I é igual a $\alpha$, com cada teste independente do outro, a probabilidade de se rejeitar a hipótese de igualdade dos desempenhos em termos de média, será dada pela probabilidade de se rejeitar pelo menos uma hipótese par a par. Havendo $q$ testes par a par, a probabilidade de se rejeitar a hipótese de igualdade entre todas as carteiras simultaneamente, sendo a hipótese verdadeira, será dada por: $\alpha^{*}=1-(1-\alpha)^{q}$

No presente caso, como são 5 carteiras para cada modelo, tem-se:

$$
\alpha^{*}=1-(1-0,05)^{4} \cong 18,55 \%
$$

Tendo presente que também se quer determinar se são significantes as diferenças de desempenho entre as carteiras otimizadas, uma carteira com diversificação ingênua e o Ibovespa, idêntico raciocínio pode ser utilizado. Todavia, neste caso, o procedimento inflaria ainda mais a probabilidade de rejeição da hipótese de igualdade, sendo esta verdadeira, dado que seriam 7 carteiras (5 otimizadas, I com diversificação ingênua e o Ibovespa), com $\alpha^{*} \cong 26,49 \%$.

Em decorrência da inadequação dos testes $t$ e $z$ para estas análises, adotase outras técnicas de análise, adequadas para comparações múltiplas. Quando um destes testes indicar a existência de diferença significativa, depreende-se que pelo menos uma das médias é diferente das demais. Neste caso quer se saber qual(is) é(são) diferente(s). Para tal, procede-se como descrito em Ferraz e Taciro Júnior (2000). O procedimento é chamado de step-down, e é o seguinte: inicialmente, testa-se a hipótese de igualdade para todas as carteiras da amostra, é o teste chamado de nível I. Se a hipótese de igualdade for aceita interrompe-se o processo. Sendo a hipótese rejeitada, afirma-se que ao menos uma das médias tem diferença significativa em relação às demais. Procede-se então, ao teste de nível 2, onde são comparadas todas as combinações de carteiras, menos uma carteira, ou seja, uma combinação de 5,4 a 4, ou de 7,6 a 6 , conforme o caso. Caso não se rejeite a hipótese nula para alguma combinação, assume-se que todas as combinações de carteiras descendentes deste grupo são "não significantes por implicação", não sendo efetuados os testes correspondentes, por óbvio. Caso seja rejeitada a hipótese de homogeneidade para qualquer grupo, é necessário passar para o nível seguinte, agora uma combinação de 5,3 a 3, ou de 7, 5 a 5, e repetir os procedimentos, sucessivamente.

Outra vez os testes são divididos em dois procedimentos: um para testar eventuais diferenças entre as médias dos retornos, e outro para determinar a existência de eventual diferença entre os Índices de Sharpe das carteiras, dadas às características específicas da distribuição destes últimos. 
bi) Comparação dos desempenhos das carteiras pelas suas médias de retornos: no primeiro procedimento, para testar a existência de eventuais diferenças entre as médias dos retornos, adota-se a técnica de análise da variância - ANOVA. De acordo com Stevenson (I98I), aplica-se a análise da variância para determinar se as médias de duas ou mais populações são iguais. No caso do teste estatístico levar à aceitação da hipótese de nulidade, conclui-se que as diferenças observadas entre as médias amostrais são casuais, ou seja, devidas a variações aleatórias na amostra. Quando há a rejeição da hipótese de nulidade, conclui-se que as diferenças entre as médias amostrais são muito grandes para serem creditadas unicamente ao acaso.

Uma vez que o rigor estatístico faz algumas exigências para a aplicação do teste de ANOVA, não totalmente satisfeitas aqui, quais sejam: I) as amostras devem ser aleatórias e independentes (por acessibilidade trabalhou-se com os vinte principais ativos do Ibovespa); 2) as amostras devem ser extraídas de populações Normais; e, 3) as populações devem ter variâncias iguais; aplica-se, ainda, um outro teste não paramétrico, que não exige tais hipóteses, exigindo apenas que a variável básica tenha distribuição contínua, que é o teste de análise da variância de um critério de Kruskal-Wallis. Stevenson (I98I) descreve o processo. Trata-se todas as observações como sendo pertencentes a uma única amostra. Cada observação é convertida em um posto, sendo atribuído o posto I para o menor valor, o posto 2 para o valor subseqüente e assim sucessivamente. A estatística teste $H$ é calculada pela fórmula:

$$
H=\frac{12}{N(N+1)} \sum_{j=1}^{k} \frac{\left(R_{j}\right)^{2}}{n_{j}}-3(N+1)
$$

Onde: $\quad N$ é o número total de observações;

$k$ é o número de amostras;

$n_{j}$ é o número de observações na j-ésina amostra;

$R_{j}$ é a soma dos postos da j-ésima amostra.

A estatística $H$ tem distribuição do tipo qui-quadrado $\left(\chi^{2}\right)$ com $k$-1 graus de liberdade. Logo, o valor de $H$ calculado pode ser comparado com valores tabulados de qui-quadrado, sendo a hipótese nula rejeitada se o valor calculado for maior que o valor crítico tabulado, considerado o nível de significância escolhido.

b2) Comparação dos desempenhos das carteiras pelos seus Índices de Sharpe: o segundo procedimento, para testar a eventual diferença de desempenho entre os Índices de Sharpe das carteiras, é também realizado a partir de um teste específico, proposto por Jobson e Korkie (I98I), e que leva em consideração as características específicas da distribuição dos retornos do Índice de Sharpe amostral. Este é um teste do tipo Wald, que usa as diferenças transformadas do Índice de Sharpe, para realizar um teste assintótico múltiplo de $n$ carteiras, cujas características são demonstradas a seguir. 
Os estimadores utilizados para o teste são: o vetor das diferenças transformadas dos índices de Sharpe $(S \hat{h})$ e a matriz de covariâncias $(\Theta)$ entre os retornos excessivos das carteiras. $O$ vetor das diferenças transformadas tem $n-1$ elementos, com cada elemento dado por:

$$
S h_{\text {in }}=\left(\sigma_{n} \mu_{i}-\sigma_{i} \mu_{n}\right), \operatorname{com} i=1,2, \ldots, n-1 \text { (I5) }
$$

Por sua vez, cada elemento da matriz de covariâncias é dado por:

$$
\begin{aligned}
& \theta_{i j}=\frac{1}{T}\left[\sigma_{n}^{2} \sigma_{i} \sigma_{j}-\sigma_{j n} \sigma_{n} \sigma_{i}-\sigma_{i n} \sigma_{n} \sigma_{j}+\sigma_{n}^{2} \sigma_{i j}+\frac{1}{2} \mu_{i} \mu_{j} \sigma_{n}^{2}-\frac{\mu_{n} \mu_{j}}{4 \sigma_{n} \sigma_{i}}\left(\sigma_{i n}^{2}+\sigma_{i}^{2} \sigma_{n}^{2}\right)\right. \\
& \left.-\frac{\mu_{n} \mu_{i}}{4 \sigma_{n} \sigma_{j}}\left(\sigma_{j n}^{2}+\sigma_{j}^{2} \sigma_{n}^{2}\right)+\frac{\mu_{n}^{2}}{4 \sigma_{i} \sigma_{j}}\left(\sigma_{i j}^{2}+\sigma_{i}^{2} \sigma_{j}^{2}\right)\right]
\end{aligned}
$$

Esta é uma matriz quadrada com $n-1$ linhas por $n-1$ colunas. Sua inversa é denotada por $\left(\Theta^{-1}\right)$. A estatística teste, do tipo Wald, demonstrada em Greene (I997), é dada por: $S \hat{h}^{\prime} \Theta^{-1} S \hat{h}(I 7)$

Seu resultado pode então ser comparado com uma distribuição do $\chi^{2}$ padronizada. Quando a estatística teste for maior do que a estatística $\chi^{2}$ crítica, rejeitase a hipótese de igualdade dos Índices de Sharpe, e efetua-se o procedimento de testes em cascata do tipo step-down, já descrito.

As Figuras I, 2, 3, 4, 5 e 6, a seguir, mostram um resumo dos procedimentos metodológicos utilizados.

\section{FIGURA I}

DETERMINAÇÃO DAS CARTEIRAS, TESTE DE NORMALIDADE E CÁLCULO DAS ESTATÍSTICAS BÁSICAS

Passo I Coleta de dados de retornos históricos de todos os ativos, da TMS, do Ibovespa e do IGP-DI.

Passo 2 Determinação da composição das carteiras otimizadas com o uso dos modelos de Markowitz e de Sharpe e auxílio do recurso Solver, do Excel. Determinação da composição da carteira com diversificação ingênua.

Passo 3 Determinação dos retornos de todas as carteiras, desde julho/I995 até junho/2000.

Passo 4 Teste preliminar: teste de normalidade de Kolmogorov-Smirnov, para determinação se as populações têm distribuição Normal.

Passo 5 Cálculo das estatísticas básicas: médias aritmética e geométrica, desvios padrão e variâncias. Cálculo dos Índices de Sharpe de todas as carteiras. 


\section{FIGURA 2}

TESTES $T$ - COMPARAÇÃO DO DESEMPENHO MARKOWITZ X SHARPE MEDIDO PELOS RETORNOS DAS CARTEIRAS

Teste $\mathrm{t}$

\begin{tabular}{ccc}
\hline \multicolumn{2}{c}{ Verificação de diferença de médias de retornos das carteiras, conforme a seguir: } \\
\hline Markowitz I8 & versus & Sharpe I8 \\
\hline Markowitz I5 & versus & Sharpe I5 \\
\hline Markowitz I2 & versus & Sharpe I2 \\
\hline Markowitz 9 & versus & Sharpe 9 \\
\hline Markowitz 6 & versus & Sharpe 6 \\
\hline $\begin{array}{l}\text { Conjunto das 5 médias obtidas } \\
\text { com as carteiras de Markowitz }\end{array}$ & versus & $\begin{array}{c}\text { Conjunto das 5 médias obtidas } \\
\text { com as carteiras de Sharpe }\end{array}$ \\
\hline
\end{tabular}

\section{FIgURA 3}

TESTES Z - COMPARAÇÃO DO DESEMPENHO MARKOWITZ X SHARPE MEDIDO PELO ÍNDICE DE SHARPE

\section{Teste $\mathrm{z}$}

Verificação de diferença de médias de Índices de Sharpe das carteiras, conforme a seguir:

\begin{tabular}{ccc}
\hline Markowitz I8 & versus & Sharpe I8 \\
\hline Markowitz I5 & versus & Sharpe I5 \\
\hline Markowitz I2 & versus & Sharpe I2 \\
\hline Markowitz 9 & versus & Sharpe 9 \\
\hline Markowitz 6 & versus & Sharpe 6 \\
\hline $\begin{array}{c}\text { Conjunto das 5 médias dos } \\
\text { as carteiras de Markowitz }\end{array}$ & versus & $\begin{array}{c}\text { Conjunto das 5 médias dos } \\
\text { Índices de Sharpe obtidas com } \\
\text { as carteiras de Sharpe }\end{array}$ \\
\hline
\end{tabular}




\section{FIGURA 4}

TESTES $F$ - ANOVA - COMPARAÇÃO MÚLTIPLA DE DESEMPENHO CONFORME O PRAZO UTILIZADO PARA A OTIMIZAÇÃO, CARTEIRA DE MERCADO E CARTEIRA COM DIVERSIFICAÇÃO INGÊNUA - MEDIÇÃO DE DESEMPENHO PELOS RETORNOS MÉDIOS OBTIDOS

\section{Teste F - ANOVA}

\section{Comparação múltipla para verificação da diferença de médias} entre os retornos das carteiras, conforme a seguir:

Cinco carteiras otimizadas com o modelo de Markowitz.

Cinco carteiras otimizadas com o modelo de Sharpe.

Cinco carteiras otimizadas com o modelo de Markowitz + carteira com diversificação ingênua + Ibovespa.

Cinco carteiras otimizadas com o modelo de Sharpe + carteira com diversificação ingênua + Ibovespa.

\section{FIGURA 5}

TESTES KRUSKAL-WALLIS - COMPARAÇÃO MÚLTIPLA DE DESEMPENHO CONFORME O PRAZO UTILIZADO PARA A OTIMIZAÇÃO, CARTEIRA DE MERCADO E CARTEIRA COM DIVERSIFICAÇÃO INGÊNUA - MEDIÇÃO DE DESEMPENHO PELOS RETORNOS MÉDIOS OBTIDOS

Análise da Variância de um Critério de Kruskal-Wallis

Comparação múltipla para verificação da diferença de médias entre os retornos das carteiras, conforme a seguir:

Cinco carteiras otimizadas com o modelo de Markowitz.

Cinco carteiras otimizadas com o modelo de Sharpe.

Cinco carteiras otimizadas com o modelo de Markowitz + carteira com diversificação ingênua + Ibovespa.

Cinco carteiras otimizadas com o modelo de Sharpe + carteira com diversificação ingênua + Ibovespa. 


\section{FIGURA 6}

TESTES WALD - COMPARAÇÃO MÚLTIPLA DE DESEMPENHO CONFORME O PRAZO UTILIZADO PARA A OTIMIZAÇÃO, CARTEIRA DE MERCADO E CARTEIRA COM DIVERSIFICAÇÃO INGÊNUA - MEDIÇÃO DE DESEMPENHO PELO ÍNDICE DE SHARPE DAS CARTEIRAS

Teste de Wald - diferenças transformadas dos Índices de Sharpe

Comparação múltipla para verificação da diferença de médias dos Índices de Sharpe, conforme a seguir:

Cinco carteiras otimizadas com o modelo de Markowitz.

Cinco carteiras otimizadas com o modelo de Sharpe.

Cinco carteiras otimizadas com o modelo de Markowitz + carteira com diversificação ingênua + Ibovespa.

Cinco carteiras otimizadas com o modelo de Sharpe + carteira com diversificação ingênua + Ibovespa.

Entende-se necessário destacar que, pelo procedimento descrito anteriormente, quando realizado um teste de comparação múltipla com sete carteiras, em qualquer dos casos, caso o teste não possa rejeitar a hipótese de homogeneidade, nem seria necessário realizar testes dos subconjuntos daqueles, uma vez que por implicação os subconjuntos também são homogêneos. O presente procedimento metodológico faz questão de incluir um teste com as cinco carteiras otimizadas de acordo com cada modelo apenas para efeito didático.

\section{RESULtAdos}

\subsection{TESTE DE NORMALIDADE}

Para testar se as amostras utilizadas provêm de populações com distribuição Normal (Ho) ou não (HI), realizou-se o teste de Kolmogorov-Smirnov, cujos resultados são apresentados na Tabela I, a seguir: 


\section{TABELA I}

TESTE DE NORMALIDADE DA AMOSTRA KOLMOGOROV-SMIRNOV

\begin{tabular}{|c|c|c|c|c|}
\hline Amostra & $\begin{array}{c}z \text { teste } \\
\text { Kolmogorov-Smirnov }\end{array}$ & z crítico & Resultado & Comentário \\
\hline Sharpe I8 meses & 0,680 & 1,960 & Não significante & aceita-se Ho \\
\hline Sharpe I5 meses & 0,663 & 1,960 & Não significante & aceita-se Ho \\
\hline Sharpe I2 meses & 0,729 & 1,960 & Não significante & aceita-se Ho \\
\hline Sharpe og meses & 0,856 & 1,960 & Não significante & aceita-se Ho \\
\hline Sharpe o 6 meses & 0,785 & 1,960 & Não significante & aceita-se Ho \\
\hline Markowitz I8 meses & 0,889 & 1,960 & Não significante & aceita-se Ho \\
\hline Markowitz I5 meses & $\mathrm{I}, \mathrm{I} 7 \mathrm{O}$ & 1,960 & Não significante & aceita-se Ho \\
\hline Markowitz I2 meses & 0,930 & 1,960 & Não significante & aceita-se Ho \\
\hline Markowitz o९ meses & 0,901 & $I, 960$ & Não significante & aceita-se Ho \\
\hline Markowitz o6 meses & 0,666 & 1,960 & Não significante & aceita-se Ho \\
\hline Ibovespa & $\mathrm{I}, 080$ & 1,960 & Não significante & aceita-se Ho \\
\hline Ingênua & 0,870 & 1,960 & Não significante & aceita-se Ho \\
\hline
\end{tabular}

Todos os resultados aceitam a hipótese de que as populações são aproximadamente Normais.

\subsection{ESTATÍSTICAS BÁSICAS}

A partir dos resultados dos retornos de cada carteira, procedeu-se ao cálculo das chamadas estatísticas básicas, englobando: médias aritméticas e geométricas, desvios padrão, Índices de Sharpe e seus desvios padrão, apresentados a seguir, na Tabela 2: 


\section{TABELA 2}

\section{ESTATÍSTICAS BÂSICAS}

\begin{tabular}{|c|c|c|c|c|c|}
\hline Amostras & $\begin{array}{c}\text { Média } \\
\text { Geométrica }\end{array}$ & $\begin{array}{c}\text { Média } \\
\text { Aritmética }\end{array}$ & $\begin{array}{l}\text { Desvio } \\
\text { Padrão }\end{array}$ & $\begin{array}{c}\text { Índice de } \\
\text { Sharpe }\end{array}$ & $\begin{array}{c}\text { Desvio Padrão } \\
\text { IS }\end{array}$ \\
\hline Sharpe I8 meses & 0,886 & $\mathrm{I}, 524$ & II,O70 & 0,018 & I,OI7 \\
\hline Sharpe I5 meses & 0,865 & $\mathrm{I}, 53 \mathrm{I}$ & II, 348 & 0,018 & $\mathrm{I}, \mathrm{O} 2 \mathrm{I}$ \\
\hline Sharpe I2 meses & I,I55 & I,898 & 12,060 & 0,047 & $\mathrm{I}, \mathrm{O} 2 \mathrm{I}$ \\
\hline Sharpe og meses & $-0,006$ & 0,763 & $\mathrm{I} 2, \mathrm{OII}$ & $-0,047$ & $\mathrm{I}, \mathrm{O} 2 \mathrm{I}$ \\
\hline Sharpe o6 meses & 0,069 & 0,846 & 12,260 & $-0,039$ & $\mathrm{I}, \mathrm{O} 2 \mathrm{I}$ \\
\hline Markowitz I8 meses & 0,863 & $\mathrm{I}, 47 \mathrm{I}$ & 10,769 & 0,013 & $\mathrm{I}, 023$ \\
\hline Markowitz I5 meses & 0,899 & $\mathrm{I}, 580$ & $\mathrm{II}, 456$ & 0,022 & $\mathrm{I}, \mathrm{O} 2 \mathrm{I}$ \\
\hline Markowitz I2 meses & $\mathrm{I}, 034$ & $\mathrm{I}, 824$ & 12,396 & 0,040 & $\mathrm{I}, 020$ \\
\hline Markowitz o9 meses & $-0,406$ & 0,447 & $\mathrm{I} 2,664$ & $-0,070$ & $\mathrm{I}, 022$ \\
\hline Markowitz o6 meses & $-0,00 \mathrm{I}$ & 0,729 & II, 825 & $-0,05 \mathrm{I}$ & $\mathrm{I}, 023$ \\
\hline Ibovespa & $\mathrm{I}, 85 \mathrm{I}$ & 2,532 & $\mathrm{II}, 252$ & 0,107 & I,OIO \\
\hline Ingênua & 1,389 & $\mathrm{I}, 940$ & 10,302 & 0,059 & $\mathrm{I}, \mathrm{OI} 4$ \\
\hline
\end{tabular}

Uma ordenação simples dos resultados estabelece o seguinte ranking - Tabela 3, a seguir, para o desempenho das carteiras:

\section{TABELA 3}

RANKING DOS RESULTADOS MÉDIOS ARITMÉTICOS DE TODAS AS CARTEIRAS

\begin{tabular}{|c|c|c|c|c|c|}
\hline Ranking & Carteira & Resultado médio & Ranking & Carteira & Resultado médio \\
\hline I & Ibovespa & 2,532 & 7 & Sharpe I8 & $\mathrm{I}, 524$ \\
\hline 2 & Ingênua & $\mathrm{I}, 940$ & 8 & Markowitz I8 & $\mathrm{I}, 47 \mathrm{I}$ \\
\hline 3 & Sharpe I2 & 1,898 & 9 & Sharpe 6 & 0,846 \\
\hline 4 & Markowitz I2 & $\mathrm{I}, 824$ & IO & Sharpe 9 & 0,763 \\
\hline 5 & Markowitz I5 & $\mathrm{I}, 580$ & II & Markowitz 6 & 0,729 \\
\hline 6 & Sharpe I5 & $\mathrm{I}, 53 \mathrm{I}$ & I2 & Markowitz 9 & 0,447 \\
\hline
\end{tabular}

Em termos nominais, os desempenhos das carteiras otimizadas foram inferiores aos resultados alcançados pelo Ibovespa e pela carteira com diversificação ingênua. Pode-se destacar um padrão formado pelo desempenho conforme os prazos tomados como base para otimização, apresentado na Tabela 4, a seguir: 


\section{TABELA 4}

PADRÃO DE DESEMPENHO CONFORME O PRAZO UTILIZADO PARA A OTIMIZAÇÃO

\begin{tabular}{ccccc}
\hline Ranking & $\begin{array}{c}\text { Sharpe/ } \\
\text { prazo }\end{array}$ & $\begin{array}{c}\text { Resultado } \\
\text { Sharpe }\end{array}$ & $\begin{array}{c}\text { Markowitz/ } \\
\text { Prazo }\end{array}$ & $\begin{array}{c}\text { Resultado } \\
\text { Markowitz }\end{array}$ \\
\hline I & I2 meses & I,898 & I2 meses & I,824 \\
\hline 2 & I5 meses & I,53I & I5 meses & I,580 \\
\hline 3 & I8 meses & I,524 & I8 meses & I,47I \\
\hline 4 & 06 meses & 0,846 & 06 meses & 0,729 \\
\hline 5 & 09 meses & 0,763 & 09 meses & 0,447 \\
\hline
\end{tabular}

Observa-se que o desempenho obtido pelo MIU foi superior ao obtido com o MM em quatro dos cinco prazos utilizados. Resta determinar se as diferenças encontradas são significativas. Isto é determinado a seguir com os testes $t$, e $z$.

\subsection{TESTES T - DIFERENÇAS DE MÉDIAS DE RETORNOS E TESTES Z - DIFERENÇAS DE ÍNDICES DE SHARPE - MARKOWITZ VERSUS SHARPE}

Já descritos na seção dedicada à metodologia da pesquisa, passa-se diretamente aos resultados obtidos, lembrando sempre que a hipótese nula pressupõe igualdade de médias, enquanto a hipótese alternativa indica diferença significativa de médias. Os resultados estão explicitados nas Tabelas 5 e 6 , a seguir.

\section{TABELA 5}

DIFERENÇA DE MÉDIAS DE RETORNOS SHARPE X MARKOWITZ

\begin{tabular}{|c|c|c|c|c|}
\hline & \\
\hline & $t$ teste & $t$ crítico & Resultado & Comentário \\
\hline Sharpe x Markowitz I8 meses & $0, \mathrm{II} 4$ & $2, \mathrm{OOI}$ & não significante & aceita-se Ho \\
\hline Sharpe x Markowitz I5 meses & $-0, \mathrm{I} 25$ & 2,001 & não significante & aceita-se Ho \\
\hline Sharpe x Markowitz I2 meses & O,I9I & 2,001 & não significante & aceita-se Ho \\
\hline Sharpe x Markowitz og meses & 0,710 & 2,001 & não significante & aceita-se Ho \\
\hline Sharpe x Markowitz o6 meses & 0,190 & 2,001 & não significante & aceita-se Ho \\
\hline Sharpe x Markowitz geral & 1,700 & 2,777 & não significante & aceita-se Ho \\
\hline
\end{tabular}




\section{TABELA 6}

DIFERENÇA DE MÉDIAS DE ÍNDICES DE SHARPE SHARPE X MARKOWITZ

\begin{tabular}{lcccc} 
& $z$ teste & $z$ crítico & Resultado & Comentário \\
\hline Sharpe x Markowitz I8 meses & 0,107 & I,960 & não significante & aceita-se Ho \\
\hline Sharpe x Markowitz I5 meses & $-0,120$ & I,960 & não significante & aceita-se Ho \\
\hline Sharpe x Markowitz I2 meses & 0,230 & I,960 & não significante & aceita-se Ho \\
\hline Sharpe x Markowitz o9 meses & 0,636 & I,960 & não significante & aceita-se Ho \\
\hline Sharpe x Markowitz o6 meses & 0,221 & I,960 & não significante & aceita-se Ho \\
\hline Sharpe x Markowitz geral & I,896 & I,960 & não significante & aceita-se Ho \\
\hline
\end{tabular}

A aceitação da hipótese nula significa que, em um nível de confiança de $95 \%$, não há diferença de desempenho entre a aplicaçao de um e outro modelo, seja a comparação realizada pelas médias de retornos obtidos, seja por meio da comparaçao dos índices de Sharpe das carteiras.

\subsection{TESTES F - COMPARAÇÃO MÚLTIPLA DE MÉDIAS DE RETORNOS}

Relembrando que, além da comparação dos desempenhos obtidos pelas carteiras otimizadas de acordo com o MM e com o MIU, outro objetivo do estudo era a comparação dos desempenhos obtidos por cada uma das Teorias, variando-se as janelas de dados históricos, ou seja, estabelecendo-se prazos diferentes como base para a otimização das carteiras. Também era objetivo comparar estes desempenhos, de cada uma das Teorias, com o desempenho das carteiras de mercado - aqui considerado o Ibovespa, e de uma carteira com diversificação ingênua. As Tabelas 7 e 8, a seguir demonstram os resultados encontrados:

\section{TABELA 7}

TESTE F COMPARAÇÃO MÚLTIPLA DE RETORNOS:

CARTEIRAS OTIMIZADAS

\begin{tabular}{lcccc} 
& $\boldsymbol{F}$ teste & $\boldsymbol{F}$ crítico & Resultado & Comentário \\
\hline Cinco medias Markowitz & $0, \mathrm{I} 49$ & 2,400 & não significante & aceita-se Ho \\
\hline Cinco medias Sharpe & $0, \mathrm{I04}$ & 2,400 & não significante & aceita-se Ho \\
\hline
\end{tabular}




\section{TABELA 8}

TESTE F COMPARAÇÃO MÚLTIPLA DE RETORNOS:

CARTEIRAS OTIMIZADAS, CARTEIRA DE MERCADO E CARTEIRA COM DIVERSIFICAÇÃO INGÊNUA

\begin{tabular}{lcccc} 
& $\boldsymbol{F}$ teste & $\boldsymbol{F}$ crítico & Resultado & Comentário \\
\hline $\begin{array}{l}\text { Cinco medias Markowitz }+ \\
\text { Ibovespa + Ingênua }\end{array}$ & $0,23 \mathrm{I}$ & $2, \mathrm{I} 20$ & não significante & aceita-se Ho \\
\hline $\begin{array}{l}\text { Cinco medias Sharpe }+ \\
\text { Ibovespa + Ingênua }\end{array}$ & $0, \mathrm{I7} 8$ & $2, \mathrm{I} 20$ & não significante & aceita-se Ho \\
\hline
\end{tabular}

Outra vez, não são observadas diferenças significativas de desempenho entre os resultados obtidos utilizando um ou outro prazo de otimização de carteiras. Isto significa que, do ponto de vista estatístico, nenhum dos prazos utilizados para a otimização das carteiras é superior aos demais. A inclusão das carteiras Ibovespa e Ingênua mantém o resultado. Como observado, não havendo média(s) diferente(s), os testes são encerrados sem a análise de subgrupos, uma vez que todos os subgrupos estão contidos no grupo maior.

\subsection{COMPARAÇÃO MÚLTIPLA DE MÉdIAS DE RETORNOS - KRUSKAL-WALLIS}

Para confirmação dos resultados anteriores, do teste $F$, foi também realizado um teste de comparação múltipla por um critério de Kruskal-Wallis. Todos os resultados estão demonstrados nas Tabelas 9 e ıо, a seguir:

\section{TABELA 9}

KRUSKAL-WALLIS - TESTE H PARA MÉDIAS DE RETORNOS DAS CARTEIRAS OTIMIZADAS

\begin{tabular}{lcccc} 
& $\boldsymbol{H}$ teste $-\boldsymbol{\chi}^{\mathbf{2}}$ & $\boldsymbol{H}$ crítico $-\boldsymbol{\chi}^{\mathbf{2}}$ & Resultado & Comentário \\
\hline Cinco medias Markowitz & 0,725 & 9,488 & não significante & aceita-se Ho \\
\hline Cinco medias Sharpe & 0,627 & 9,488 & não significante & aceita-se Ho \\
\hline
\end{tabular}




\section{TABELA IO}

KRUSKAL-WALLIS - TESTE $H$ PARA MÉDIAS DOS RETORNOS DAS CARTEIRAS OTIMIZADAS, DA CARTEIRA DE MERCADO E DA CARTEIRA COM DIVERSIFICAÇÃO INGÊNUA

\begin{tabular}{lcccc} 
& $\boldsymbol{H}$ teste $-\chi^{2}$ & $H$ crítico $-\chi^{2}$ & Resultado & Comentário \\
\hline $\begin{array}{l}\text { Cinco medias Markowitz }+ \\
\text { Ibovespa + Ingênua }\end{array}$ & 2,977 & I2,592 & não significante & $\begin{array}{c}\text { aceita-se } \\
\text { Ho }\end{array}$ \\
\hline $\begin{array}{l}\text { Cinco medias Sharpe }+ \\
\text { Ibovespa + Ingênua }\end{array}$ & 3,049 & I2,592 & não significante & $\begin{array}{c}\text { aceita-se } \\
\text { Ho }\end{array}$ \\
\hline
\end{tabular}

Também estes resultados levam à aceitação da hipótese nula, ou seja, de que as diferenças entre as médias são não significantes. Não há a necessidade de seguir com a realização de testes para os subgrupos e os testes são interrompidos.

\subsection{TESTES WALD - COMPARAÇÃO MÚLTIPLA DE ÍNDICES DE SHARPE}

\section{TABELA I I}

TESTES WALD - COMPARAÇÃO MÚLTIPLA DE ÍNDICES DE SHARPE: CARTEIRAS OTIMIZADAS

\begin{tabular}{lcccc} 
& $\chi^{2}$ teste & $\chi^{2}$ crítico & Resultado & Comentário \\
\hline Cinco médias Markowitz & 7,594 & 9,488 & não significante & aceita-se Ho \\
\hline Cinco médias Sharpe & 8,086 & 9,488 & não significante & aceita-se Ho \\
\hline
\end{tabular}

\section{TABELA I 2}

COMPARAÇÃO MÚLTIPLA DE ÍNDICES DE SHARPE: CARTEIRAS OTIMIZADAS, CARTEIRA DE MERCADO E CARTEIRA COM DIVERSIFICAÇÃO INGÊNUA

\begin{tabular}{lcccc} 
& $\chi^{2}$ teste & $\chi^{2}$ crítico & Resultado & Comentário \\
\hline $\begin{array}{l}\text { Cinco médias Markowitz + } \\
\text { Ibovespa + Ingênua }\end{array}$ & 9,732 & I2,592 & não significante & $\begin{array}{c}\text { aceita-se } \\
\text { Ho }\end{array}$ \\
\hline $\begin{array}{l}\text { Cinco médias Sharpe + } \\
\text { Ibovespa + Ingênua }\end{array}$ & 10,089 & I2,592 & não significante & $\begin{array}{c}\text { aceita-se } \\
\text { Ho }\end{array}$ \\
\hline
\end{tabular}

Também a comparação de desempenho com a utilização do parâmetro $\mathrm{I}_{\text {sharpe }}$ mantém o resultado anterior, ou seja, nenhum dos prazos utilizados na otimizaçao das carteiras pode ser considerado superior aos demais. As diferenças entre os Índices de Sharpe encontradas devem ser creditadas a variações aleatórias da amostra. 


\section{CONCLUSÕes}

Recorde-se que o objetivo central do presente trabalho era determinar a existência de diferença significativa entre os desempenhos das Teorias de Carteira de Markowitz e de Sharpe, aplicadas na otimização de carteiras de ações no mercado brasileiro. Os resultados da pesquisa demonstram não existir evidências de superioridade no desempenho de uma em relação à outra, a despeito das simplificações adotas por Sharpe. Este resultado não deixa de evidenciar um grande mérito de Sharpe, uma vez que seu modelo, como visto, é bastante simplificado em relação àquele proposto por Markowitz.

Outro objetivo era comparar o desempenho destes modelos em relação à carteira de mercado, aqui representada pelo Ibovespa, e com uma carteira com diversificação ingênua. Esta comparaçao permitiria verificar os possíveis benefícios que a utilização destes modelos traria ao investidor. A despeito de que os testes tenham demonstrado um desempenho idêntico do ponto de vista estatístico, é interessante observar que tanto Ibovespa quanto a carteira com diversificaçao ingênua tenham obtido desempenhos nominais superiores. De qualquer forma, pode-se afirmar que não foram encontradas vantagens para o investidor na utilização dos presentes modelos como preditivos para a formação de carteiras de investimento.

Talvez este resultado possa ser creditado ao fato de que os modelos matemáticos utilizados nao "respondam" de pronto a fatos que afetam tanto positiva quanto negativamente a determinadas empresas e/ou setores econômicos. Muitas vezes, um ativo com uma boa relação histórica de risco/retorno, demora para sair da carteira otimizada, ainda que seja conhecido um fato que afetará de forma muito importante seu retorno, uma vez que o modelo é puramente matemático. A despeito de que o prazo utilizado no presente estudo seja relativamente longo, 60 meses, se pode sugerir que outros trabalhos utilizem prazos ainda mais longos. Isto pode contribuir na determinação de diferenças significativas do ponto de vista estatístico.

Finalmente, também era objetivo do trabalho determinar se algum prazo de janela móvel utilizado como base para a otimização das carteiras conforme um e outro modelo apresentava-se consistentemente superior aos demais. Também aqui, apesar de ter se formado para uma e outra teoria um padrão idêntico no rankeamento dos desempenhos das carteiras conforme o prazo utilizado, estes não podem ser considerados significativamente diferentes, devendo ser considerados casuais. Este resultado difere daquele apresentado em estudo anterior por Bruni Fama (I998). Para este ponto, também vale a sugestão do parágrafo anterior.

\section{REFERÊNCIAS}

BROCHMANN, Luciano, DRESCH, Ademar P., FIGUEIREDO, Antonio C., FRANZ, Paulo R. G. e ZANINI, Francisco A. M. A utilização da teoria de carteiras de Markowitz e do modelo de Índice 
Único de Sharpe no mercado de ações brasileiro em i999. Resenha BM\&F, São Paulo, n. I4I, p. 5I-59, set/out 2000.

BRUNI, Adriano Leal, FAMÁ, Rubens. Moderna Teoria de Portfólios: é possível captar, na prática, os benefícios decorrentes de sua utilização? Resenha BM\&F, São Paulo, n. I28, p. I9-34, nov/dez I998. CHÁRA, Alexandre Noboru, ODA, André Luiz, SENGER, Maria Carlota Morandin. Um estudo sobre diversificação na Bolsa de Valores de São Paulo. Anais em CD-ROM do 22ํㅡㄴ Encontro Nacional da Associação Nacional dos Programas de Pós-graduação em Administração - Enanpad, Foz do Iguaçu, 27-30/setembro/I998.

CHEN, S. N.; LEE, Cheng F. The sampling relationship between Sharpe's performance measure and its risk proxy: Sample size, investment horizon and market conditions. Management Science, vol. 27, n. 6, pp. 607-6ı8, June i98I.

ELLIS, Charles D. A Composição do Investimento. In BERNSTEIN, Peter L., DAMODARAN, Aswath. Administração de investimentos. Porto Alegre: Bookman, 2000.

ELTON, Edwin J., GRUBER, Martin J. Modern Portfolio Theory and Investment Analysis. 5. ed. New York: John Wiley \& Sons, Inc, I995.

ELTON, Edwin J., GRUBER, Martin J., URICH, Thomas J. Are Betas Best? The Journal of Finance, vol. XXXIII, n. 5, p. I375-1384, December, I998.

EVANS, Jonh L., ARCHER, Stephen H., Diversification and the reduction of dispersion: an empirical analysis. Journal of Finance, vol. 23, p. 76I-767, Dec. I977.

FERRAZ, Euclides M., TACIRO JÚNIOR, Affonso. Comparando o desempenho de fundos com o Índice de Sharpe. Resenha BM\&F, São Paulo, n. I42, p. 52-64, nov-dez/2000.

FISCHER, Lawrence, LORIE, James H. Some studies of variability of returns on investments in common stocks. Journal of Business, vol. 43, n. 2, p. 99-133, april I970.

GREENE, William H. Econometric Analysis. New Jersey: Prentice-Hall, I997.

HIEDA, Akinori, ODA, André Luiz. Um estudo sobre a utilização de dados históricos no modelo de Markowitz aplicado à Bolsa de Valores de São Paulo. In: Seminários de Administração, 3, Out. I998, São Paulo. Anais do III SEMEAD. São Paulo: Faculdade de Economia, Administração e Contabilidade da USP, I998.

JACOB, Nancy L. Avaliando o desempenho do investimento. Modelos de Risco. In: BERNSTEIN, Peter L., DAMODARAN, Aswath (org.). Administração de investimentos. Porto Alegre: Bookman, 2000. JOBSON, J. D.; KORKIE, Bob M. Performance Hypotesis testing with the Sharpe and Treynor measures. The Journal of Finance, September I98I, vol. XXXVI, n. 4.

MARKOWITZ, Harry. Portfolio Selection. The Journal of Finance, vol. VII, n. I, March I952.

MEYER, Paul L. Probabilidade: aplicações à estatística. Rio de Janeiro: Ao Livro Técnico S.A., I972.

SÁ, Geraldo Tosta de. Administração de investimentos, teoria de carteiras e gerenciamento do risco. Rio de Janeiro: Qualitymark, I999.

SHARPE, William F. A simplified model for portfolio analysis. Management Science, p. 277-293, I963.

STEVENSON, William J. Estatística aplicada à administração. São Paulo: Harper \& Row do Brasil, I98I.

VAUGHAN, Emmett J. Risk Management. New York: John Willey \& Sons, Inc., I997.

\section{TRAMITAÇ Ã O}

Submissão: 22/03/2004

Aprovação: 24/02/2005 
Copyright of Revista de Administração Mackenzie is the property of Universidade Presbiteriana Mackenzie, RAM-Revista de Administracao Mackenzie and its content may not be copied or emailed to multiple sites or posted to a listserv without the copyright holder's express written permission. However, users may print, download, or email articles for individual use. 